\title{
AVALIAÇÃO DA CICLAGEM DE NUTRIENTES EM ÁREAS DE REFLORESTAMENTO A PARTIR DAS FORMAS DE HUMUS
}

\author{
Fernando Pessoa $^{(\mathrm{a})}$, Fernando Cesário $^{(\mathrm{b})}$, Bruno Nascimento $^{(\mathrm{c})}$, Flávio Rosa $^{(\mathrm{c})}$ \\ (a) CEFET/RJ - campus Petrópolis / Programa de Pós-Graduação em Geografia da UFRJ, E-mail: \\ fap_rj@hotmail.com \\ (b) Programa de Pós-Graduação em Geografia da UFRJ, E-mail: fernandovieiracesario@gmail.com \\ ${ }^{(c)}$ Graduando em Geografia da UFRJ
}

\author{
Eixo: SOLOS E PAISAGENS
}

\begin{abstract}
Resumo
O ecossistema representa uma unidade complexa, na qual sistemas de forte componente biológico interagem com sistemas de natureza antrópica, o que resulta de fatores socioeconômicos, culturais e políticos que ressaltam a sua importância como unidade de avaliação da biodiversidade na escala da paisagem. Com isso, a recuperação, conservação e uso sustentável do mosaico de fragmentos existentes tornam-se fundamentais. Uma forma de avaliar o funcionamento de ecossistemas é através da utilização de indicadores que sintetizam seus dois processos fundamentais: produtividade e decomposição. Assim, o presente trabalho, desenvolvido em uma das áreas do projeto "Mutirão Reflorestamento", realizado pela Secretaria Municipal de Meio Ambiente (SMAC) do Rio de Janeiro, realizou um diagnóstico da qualidade ecossistêmica e ciclagem de nutrientes a partir da utilização de Indicadores Funcionais Globais, além de propor técnicas que facilitem o processo sucessional natural a partir do princípio da nucleação, com a transposição de solo e serapilhera.
\end{abstract}

Palavras chave: Fragmentação Florestal, Reflorestamento, Indicadores Funcionais Globais, Formas de Humus.

\section{Introdução}

O objeto deste artigo são os temas da biodiversidade e da fragmentação florestal relacionadas aos ecossistemas terrestres, que permanecem sob pressão de usos alternativos da terra e desmatamento, resultando tanto em perda de biodiversidade quanto na degradação dos serviços ecossistêmicos que afetam as comunidades nas escalas local, nacional e global.

As mudanças ambientais podem ser consideradas como uma das questões mais importantes do mundo atual. Neste contexto, destacam-se as regiões tropicais, por abrigarem as maiores taxas de biodiversidade e, ao mesmo tempo, serem as mais afetadas pelo processo de desflorestamento (Myers, 1988; 1990). 


\section{OS DESAFIOS DA GEOGRAFIA FÍSICA NA FRONTEIRA DO CONHECIMENTO \\ Instituto de Geociências - Unicamp \\ Campinas - SP \\ 28 de Junho à 02 de Julho de 2017}

No Brasil, merece destaque a Mata Atlântica, o mais afetado dos seus biomas, tendo sido reduzido a menos de 10\% de sua cobertura original (SOS Mata Atlântica e INPE, 2015). Este bioma é atualmente formado por um mosaico de fragmentos florestais de diferentes formas, tamanhos e estágios sucessionais, espalhados em meio a sistemas antrópicos, o que evidencia o caráter dinâmico da paisagem, tanto no espaço quanto no tempo. A conservação e a recuperação dos recursos naturais remanescentes da Mata Atlântica dependem, assim, de uma política de gestão dos fragmentos florestais. Porém, para se efetuar a gestão dos fragmentos florestais, é preciso conhecer não apenas o seu tamanho e forma, contiguidade a outros fragmentos e tipo de habitat matriz, mas, principalmente, o seu estado funcional. A associação do tipo de fragmento com seu histórico e com o conhecimento do funcionamento e da estrutura de seus componentes biológicos é essencial para guiar programas de conservação (Garay, 2001).

Assim, sistemas naturais, inseridos nas paisagens, identificam a unidade de avaliação da biodiversidade ao nível de ecossistema, que é uma unidade complexa na qual sistemas de forte componente biológico ecossistemas - interagem com sistemas de natureza antrópica, resultado de fatores sócio-econômicos, culturais e políticos. No bioma da Mata Atlântica, muito fragmentado e com alta diversidade de ecossistemas, a preservação do status da diversidade biológica depende da recuperação, conservação e uso sustentável do mosaico de fragmentos existentes.

Além disso, políticas e projetos que visem recuperar, manter ou até mesmo reflorestar áreas de interesse geoecológico são medidas contundentes para a proteção e manutenção da biodiversidade de biomas. $\mathrm{O}$ reflorestamento, quando executado com eficiência, é uma alternativa eficaz que abarca diversos vieses de grande importância para o meio ambiente, principalmente sob o ponto de vista geoecológico, como: melhoramento de bacias hidrográficas, recuperação de ecossistemas degradados, promoção da conectividade de fluxos e da paisagem, além de contribuir na prevenção de deslizamentos de terras e também no combate à erosão dos solos.

Para avaliação desse processo, podem ser utilizados indicadores que permitem traçar inferências sobre os processos de produção e decomposição da matéria orgânica - formas de húmus, cuja caracterização permitirá o aprofundamento do conhecimento existente sobre a influência de diferentes estágios sucessionais e em condições fisiográficas semelhantes. Pretende-se, com isso, testar a utilização de particularidades do subsistema de decomposição - diferenças das variáveis entre os sistemas - para a avaliação ambiental (Kindel 2001; Garay e Kindel, 2001; Kindel e Garay, 2001; Castro Jr, 2002). 
As formas de húmus permitem, em síntese, trabalhar interações que ocorrem no nível ecossistêmico, e não sobre espécies individuais (Ponge, 2013), o que contribui para os projetos de avaliação, restauração e manejo de fragmentos florestais da Mata Atlântica.

Por outro lado, além da avaliação, práticas de restauração devem ser ressaltadas, tendo em vista que a restauração de áreas degradadas representa uma atividade básica para a conservação in situ, refazendo comunidades e formando corredores entre fragmentos vegetacionais. Uma dessas práticas é a nucleação, que é um princípio sucessional na colonização de áreas em formação e representa uma técnica básica para as atividades antrópicas que se proponham contribuir para o reestabelecimento de comunidades, dentre as quais se destacam: transposição de solo, poleiros artificiais, transposição de galharia, entre outras (Reis et al., 2003).

Por fim, cabe destacar a importância de se problematizar a acurácia de indicadores de síntese sobre o estado funcional de fragmentos florestais e as técnicas que serão propostas de nucleação, a fim de avaliar a possibilidade de expansão de tais técnicas para outras áreas de reflorestamento.

\section{Objetivos}

Realizar, através das formas de humus, a avaliação da ciclagem de nutrientes em áreas de reflorestamento e o mapeamento das camadas orgânicas de superfície, a partir do uso de geotecnologias.

\section{Operacionalização da pesquisa}

A pesquisa foi realizada no reflorestamento do Morro da Formiga, onde foram instaladas 46 parcelas (Figura 1), a partir de um desenho amostral que contemplou o maior número de situações geoecológicas existentes na área de estudo. 


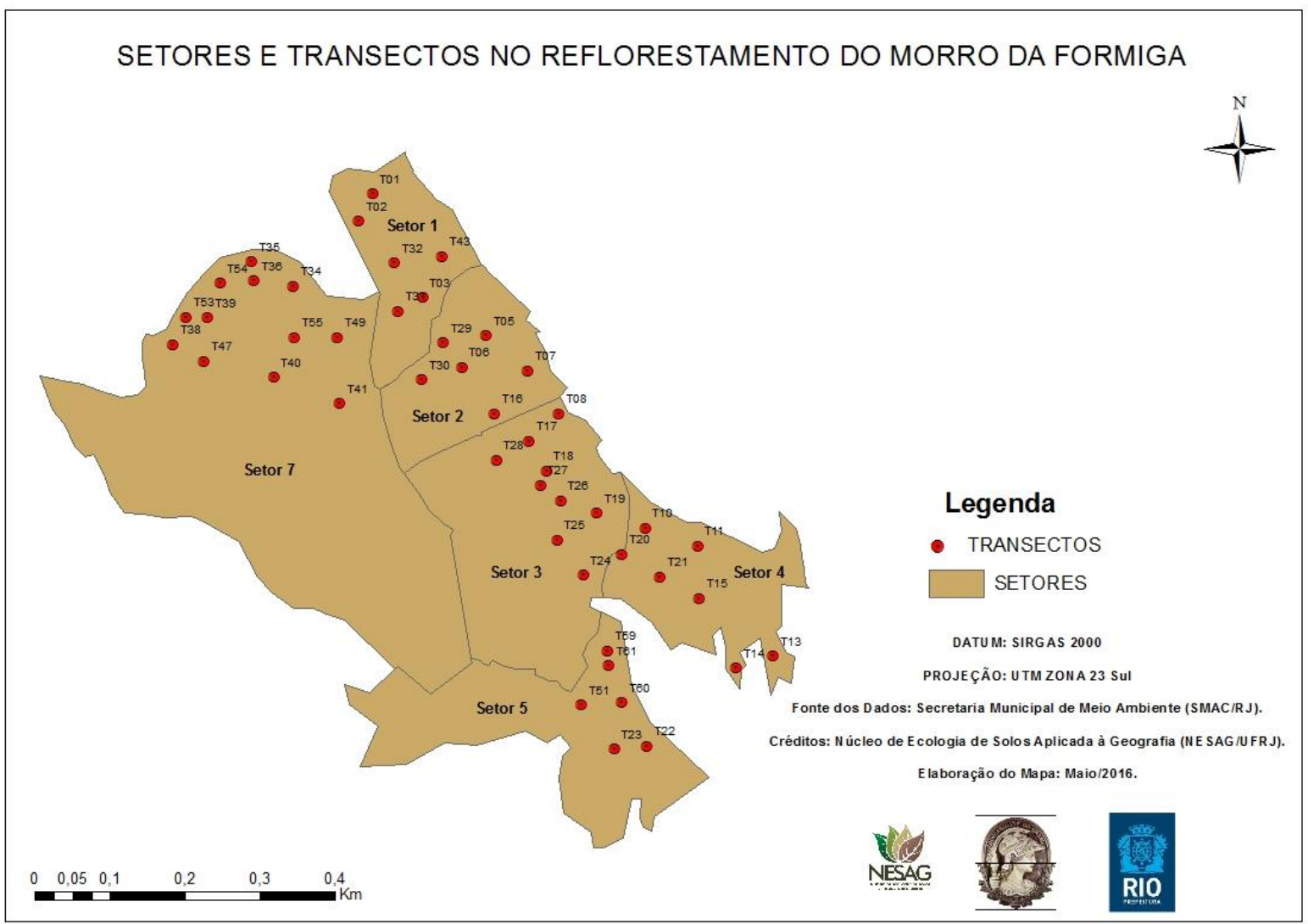

Figura 1: Disposição dos setores e transectos avaliados no reflorestamento do Morro da Formiga.

As parcelas possuem, cada uma, 10 metros de comprimento por 5 metros de largura $\left(50 \mathrm{~m}^{2}\right)$, com um coletor de produção foliar e quatro pontos de amostragem de formas de húmus em cada.

Após a instalação das parcelas, foi realizada, de acordo com a preparação, coleta e análise dos dados de produção foliar.

O material de produção foliar foi coletado com um aparato de área conhecida, intencionalmente proposto para reter as folhas que caem das árvores antes que atinja o piso florestal. Em seguida, as amostras foram acondicionadas e levadas para laboratório, onde foram secas a $55^{\circ} \mathrm{C}$ durante 48 horas e separadas em folhas, material lenhoso e refugo.

As amostras de Forma de Húmus (total de 4 por parcela) foram coletadas com auxílio de aparato quadrado de ferro com $25 \mathrm{~cm}$ de lado (Figura 2). 


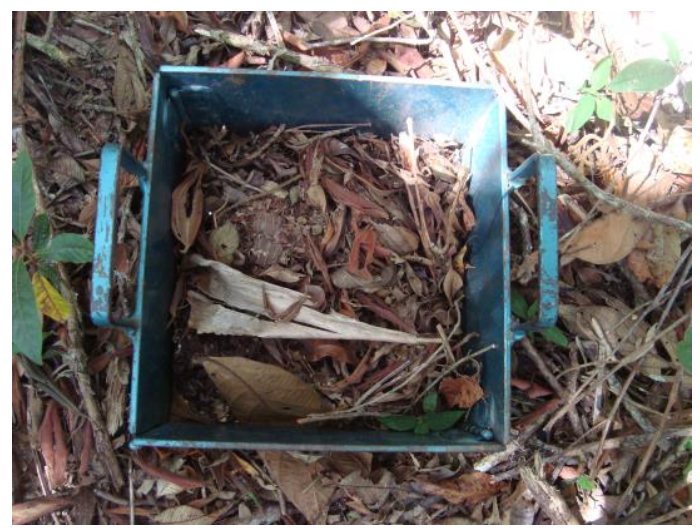

Figura 2: Quadrado de metal com 25cm de lado para coleta das Formas de Húmus (Acervo NESAG/UFRJ).

Em seguida as amostras foram acondicionadas e levadas para laboratório, onde foram secas a $55^{\circ} \mathrm{C}$ durante 48 horas e separadas em camadas que diagnosticam estágios de decomposição, são elas: Camada L: Folhas íntegras sem sinais visíveis de degradação; Camada F1: Folhas fragmentadas < 4mm; Camada F2: Folhas fragmentadas 4-2mm; BioMeso: Agregados zoogênicos < 4mm; MFB: Material Fino Bruto < 2mm; BioMeso: Agregados zoogênicos 4-2mm e Galhos (Figura 3), de acordo com (Zanella, et al., 2011).

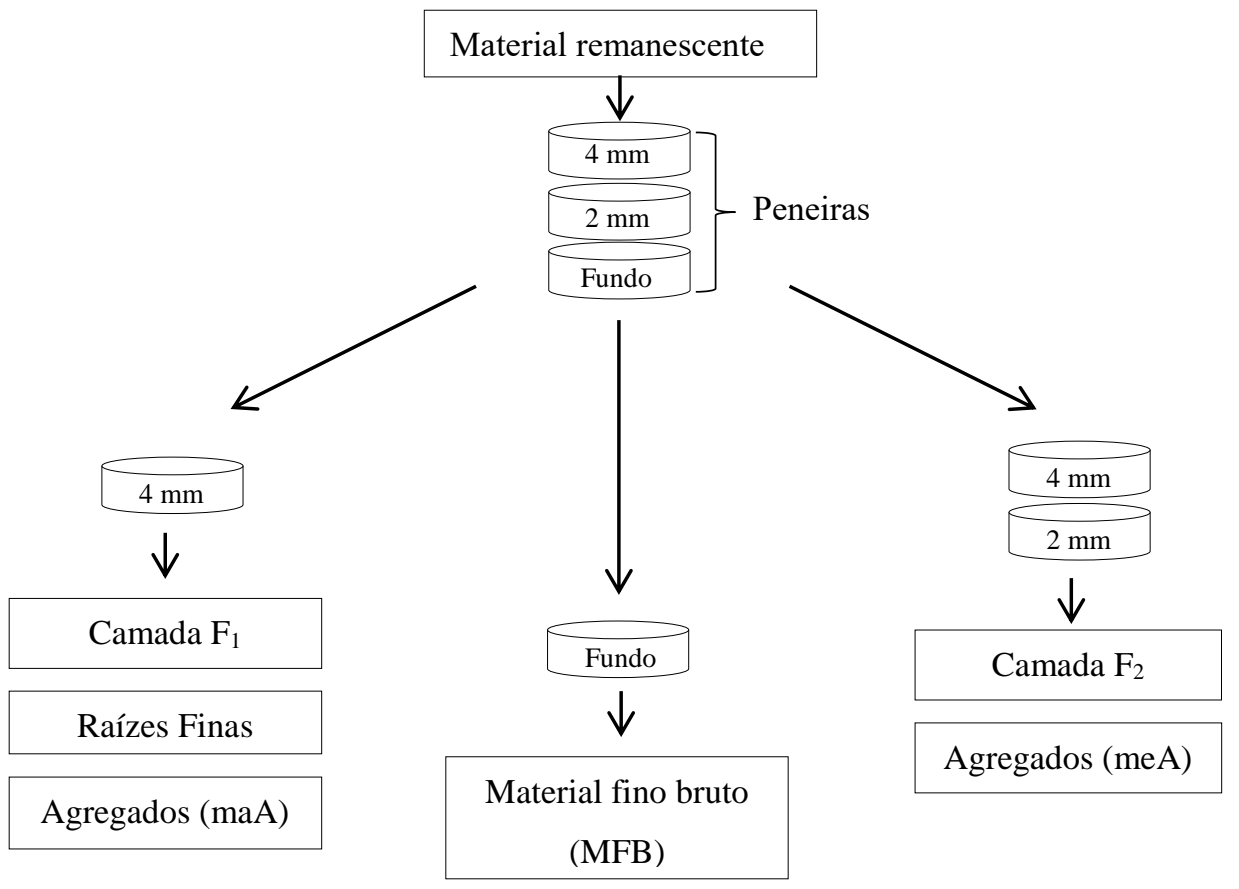


Figura 3: Processo esquemático da triagem do material remanescente das formas de humus após coleta da camada $\mathrm{L}$ em campo e da secagem $55^{\circ} \mathrm{C} / 48 \mathrm{~h}$.

\section{Resultados e Discussões}

A figura 4 apresenta os resultados da análise de variância, que tem por objetivo comparar os setores do reflorestamento, usando os dados das camadas das formas de humus.

Os resultados apontam que não há diferença significativa entre os setores no que tange aos estoques da camada L, mesmo o setor 2 apresentando médias ligeiramente maiores. No geral os estoques da camada L são baixos, o que pode evidenciar uma rápida decomposição do folhiço em todos os setores analisados ou uma baixa produção do material decíduo.

Os resultados da camada F1 seguem a mesma tendência da camada L, não apresentando diferença significativa entre os setores estudados. Esses resultados mostram que o processo de fragmentação em folhiços $>4 \mathrm{~mm}$ não apresenta diferença em todo o reflorestamento.

Os resultados da camada BioMacro apresentaram diferença significativa $(p<0,05)$ entre os tratamentos. $\mathrm{E}$ os resultados dos testes de comparações múltiplas (Tukey, $p<0,05$ ) mostraram que o setor 2 difere significativamente com médias mais elevadas dos setores 5 e 7 . Os setores 5 e 7 apresentaram as menores massas de agregados BioMeso entre todos os setores do reflorestamento. Esse resultado pode evidenciar tanto uma deficiência na fauna do solo desses setores, como um baixo aporte de material orgânico.

Outra camada das formas de humus que apresentou diferença significativa entre os setores do reflorestamento foi a camada F2. Nesse caso o setor 4 apresentou diferença significativa $(p<0,05)$ em relação aos setores 1, 2 e 7, apresentando estoques mais elevados para a camada F2. Ainda assim, os resultados mostram que o setor 3 também diferiu dos setores 1 e 7, e também apresentou estoques mais elevados de material orgânico fragmentado entre $4-2 \mathrm{~mm}$. O setor 5 foi o único setor que não diferiu significativamente de todos os outros setores analisados.

Por fim, a camada de galhos foi outra que também diferiu entre os setores. Os resultados apontam que o setor 3 apresentou médias maiores de galhos no solo florestal e diferiu dos setores 2 e 5 , que apresentaram as menores médias entre todos os setores. Os setores 1, 4 e 7 apresentaram médias similares entre si e não diferiram dos demais. 


\begin{tabular}{|c|c|}
\hline $\begin{array}{l}\text { XVII Simpósio Brasileiro } \\
\text { de Geografia Fisica Aplicada }\end{array}$ & $\begin{array}{l}\text { OS DESAFIOS DA GEOGRAFIA FÍSICA NA FRONTEIRA DO CONHECIMENTO } \\
\text { Instituto de Geociências - Unicamp }\end{array}$ \\
\hline $\begin{array}{l}\text { I Congresso Nacional } \\
\text { de Geografia Física }\end{array}$ & $\begin{array}{c}\text { Campinas - SP } \\
28 \text { de Junho à } 02 \text { de Julho de } 2017\end{array}$ \\
\hline
\end{tabular}



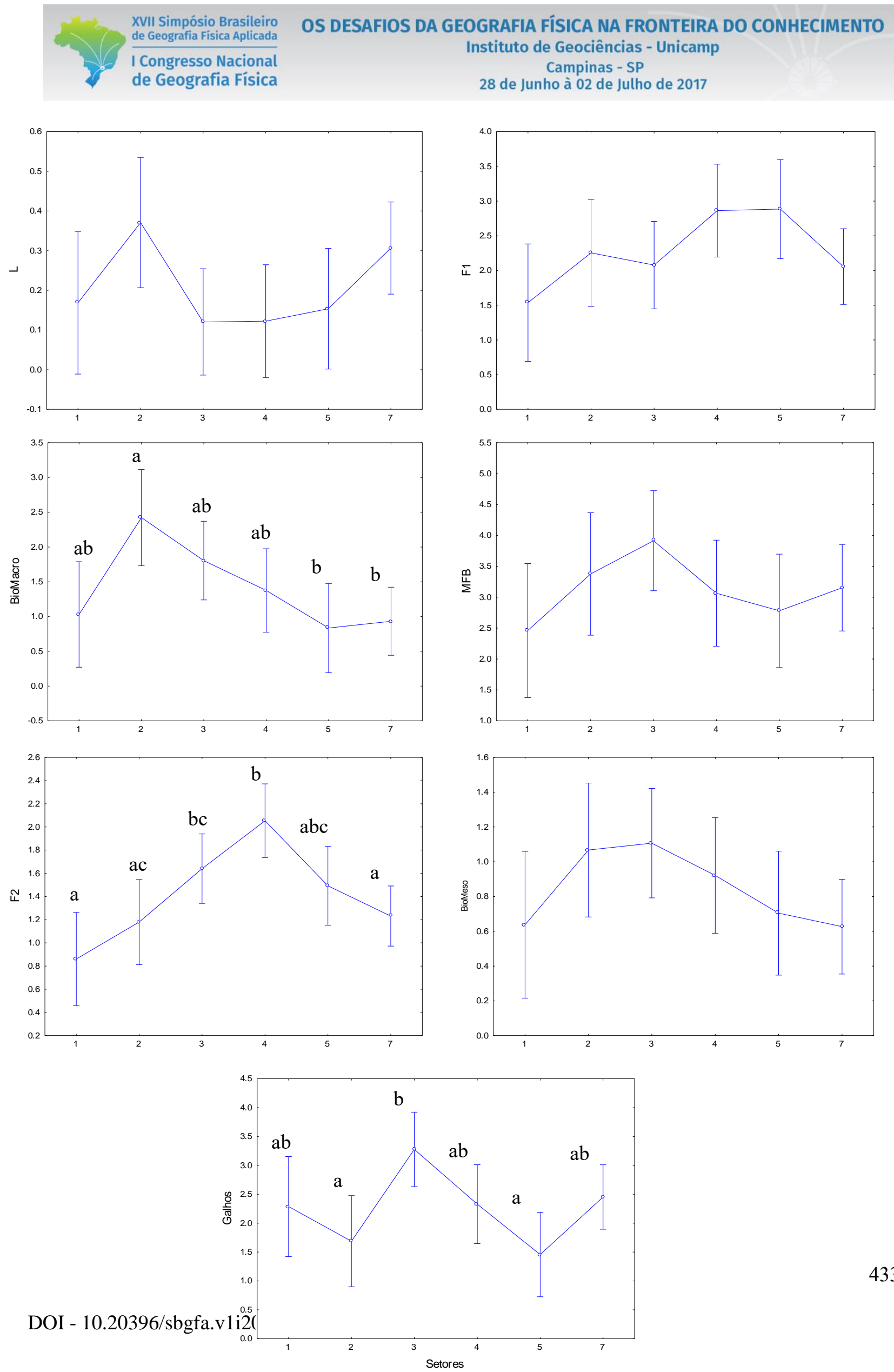
Figura 4: Comparação das estruturas das formas de humus pelo teste de análise de variância (ANOVA). Eixo das ordenadas compreendem os valores dos quadrados mínimos. Setores com diferença significativa foram analisados por teste de comparação de médias (Tukey, $\mathrm{p}<0,05$ ). Setores com a mesma letra não diferem significativamente entre si. Setores em letras representam valores não significativos (ns). Camada L: Folhas íntegras sem sinais visíveis de degradação; Camada F1: Folhas fragmentadas < 4mm; Camada F2: Folhas fragmentadas 4-2mm; BioMeso: Agregados zoogênicos < 4mm; MFB: Material Fino Bruto < 2mm; BioMeso: Agregados zoogênicos 4-2mm e Galhos.

Os resultados do teste de análise de variância (Anova) para as variáveis de produção de material decíduo entre os setores avaliados mostraram que não existe diferença significativa entre nenhum dos setores avaliados, evidenciando que a despeito de médias diferentes, a produção entre os setores não difere.

As cartas de suscetibilidade de formas de húmus (Figuras 5 e 6) possibilitaram avaliar espacialmente tanto a velocidade de decomposição quanto o potencial de incorporação de matéria orgânica nos primeiros centímetros do solo. Esses produtos também estão relacionados com a dinâmica ecossistêmica de ambientes florestais.

A relação L/F apresentada reflete a velocidade da passagem do estágio de decomposição relacionado com a lavagem para a fragmentação, essa relação possibilita avaliar o potencial de cada setor do reflorestamento em emitir $\mathrm{CO}^{2}$ através da respiração microbiana associada à respiração e a lavagem de nutrientes pela precipitação.

As cartas de susceptibilidade das formas de húmus apresentadas possibilitam uma avaliação espacial detalhada do processo de decomposição, notadamente importante para a ciclagem de nutrientes. Uma decomposição acelerada pode significar um rápido uso da necromassa orgânica inviabilizando a utilização efetiva dos nutrientes, enquanto uma decomposição lenta pode significar uma imobilização. Além disso, o produto oferece uma visão espacial do comportamento do reflorestamento no que tange a decomposição.

Em relação às cartas de suscetibilidade $\mathrm{MFB} / \mathrm{L}+\mathrm{F}$ apresentadas, esse produto possibilita avaliar a capacidade espacial do reflorestamento em incorporar matéria orgânica nos primeiros centímetros do solo. A relação $\mathrm{MFB} / \mathrm{L}+\mathrm{F}$ representa o potencial de carbono que poderá ser incorporado no solo, entretanto, deve-se ressaltar que essa avaliação deve levar em conta o tipo de solo de cada área a ser avaliada, assim como, as espécies plantadas. No geral, um maior valor da relação apresentada significa maior potencial 


\section{OS DESAFIOS DA GEOGRAFIA FÍSICA NA FRONTEIRA DO CONHECIMENTO \\ Instituto de Geociências - Unicamp \\ Campinas - SP \\ 28 de Junho à 02 de Julho de 2017}

em sequestrar carbono no solo, principalmente nos primeiros centímetros, que é o horizonte do solo mais suscetível a mudanças de uso e modificações em estrutura e compactação.

Desta maneira, as cartas temáticas apresentadas possibilitam avaliar em novos reflorestamentos com as mesmas características, o potencial de carbono que poderá ser emitido e/ou sequestrado nos primeiros centímetros dos solos. O produto dará a capacidade de planejar e entender o comportamento da dinâmica do carbono em novos reflorestamentos ou em reflorestamentos já implantados, entretanto, deve-se ressaltar que a precisão dessa estimativa é baixa (60\%), no entanto, devido à rapidez, baixo custo e replicabilidade, o produto gerado apresenta viabilidade para ser utilizado como estimativa do potencial de incorporação ou emissão carbono em áreas reflorestadas similares a área estudada.

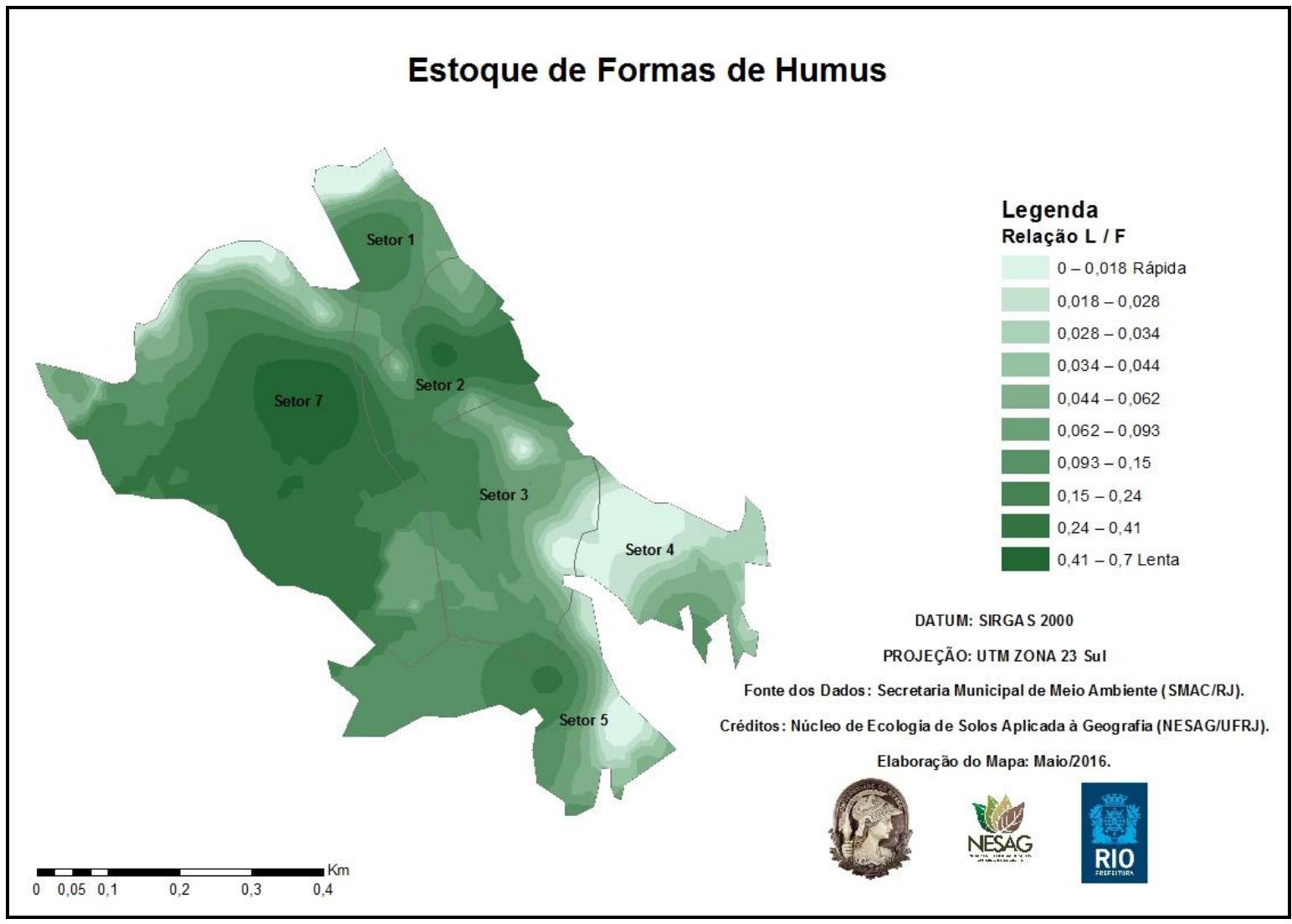

Figura 5: Carta temática da suscetibilidade das formas de húmus, relação L/F nos setores do reflorestamento. 


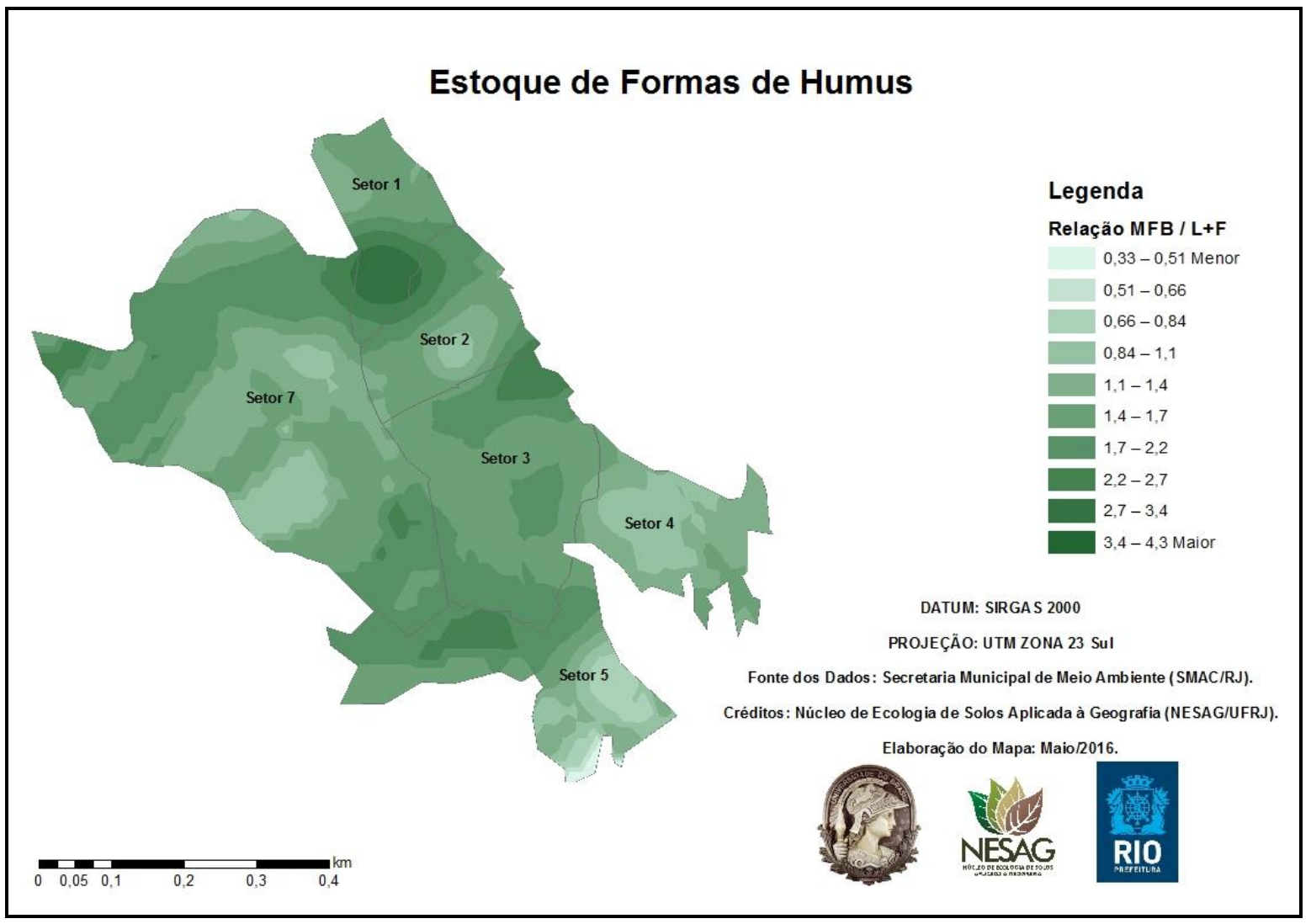

Figura 6: Carta temática da suscetibilidade das formas de húmus, relação MFB/L+F nos setores do reflorestamento.

\section{Considerações Finais}

Os dados apresentados refletem a possibilidade de avaliação de áreas reflorestadas com base nas formas de húmus, o que permite também a proposição de técnicas de restauração com base nessa metodologia.

Apesar da similaridade de algumas camadas, como a L, entre os setores, diferenças significativas foram observadas entre as camadas BioMacro, F2 e Galhos, de acordo com os resultados de análise de variância.

Cabe ressaltar que as formas de húmus, ao apresentarem uma estrutura de camadas que refletem um funcionamento - o processo de ciclagem de nutrientes, também deve ter uma análise individual das camadas, que podem se apresentar como horizontes diagnósticos nas diferenciação de áreas, como ocorreu em alguns casos nos setores do reflorestamento do Morro da Formiga, de acordo com comparação dos setores realizada por análise multivariada de ordenamento multidimensional - NMS. 
Também é importante destacar que, apesar das variáveis de produção de material decíduo não terem apresentado diferença significativa entre nenhum dos setores avaliados, foi possível observar a variabilidade desses dados entre os setores com a elaboração de cartas temáticas apresentando a distribuição espacial dessas variáveis por Krigagem Ordinária. Assim, ressalta-se a necessidade da aplicação de técnicas de geoprocessamento e estatística espacial para esses tipos de estudos.

\section{Bibliografia}

BABEL, U. Micromorphology of soil organic matter. In:GIESEKING, J.E., ed. Soil component. Berlin, SpringerVerlag, 1975. p.369-473.

BEGON, M.; HARPER, J. L.; TOWSEND, C. R. Ecology: individuals, populations and communities. Oxford Library Blackwell, 1996.

CASTRO JR, E. Valor indicador da fauna de macroartrópodes edáficos em fragmentos primários e secundários do ecossistema de florestas de tabuleiros, ES. Tese de Doutorado. Programa de Pós-Graduação em Geografia, 2002, Universidade Federal do Rio de Janeiro.

GARAY, I. Avaliação do status da biodiversidade a nível de ecossistema In: GARAY, I. \& DIAS, B. (Orgs). Conservação da biodiversidade em ecossistemas tropicais: avanços conceituais e revisão de novas metodologias de avaliação e monitoramento. Rio de Janeiro, Petrópolis: Editora Vozes, 2001.

GARAY, I. e KINDEL, A. Diversidade funcional em fragmentos de Floresta Atlântica. Valor indicador das formas de húmus florestais. In: GARAY, I. \& DIAS, B. (Orgs). Conservação da biodiversidade em ecossistemas tropicais: avanços conceituais e revisão de novas metodologias de avaliação e monitoramento. Rio de Janeiro, Petrópolis: Editora Vozes, 2001.

GARAY, I.; KINDEL, A.; JESUS, R. M. Diversity of humus forms in the Atlantic Forest ecosystems (Brazil). The Table-land Antlanic Forest. Acta Oecologia, 16, 1995, p. 553-570.

GARAY, I. \& SILVA, B. A. O. Húmus florestais: síntese e diagnóstico das interrelações vegetação/solo. Oecologia Brasiliensis, 1: 19-46, 1995.

GOLDIN, A. Reassessing the use of loss-on-ignition for estimating organic matter content in noncalcareous soils. Commun. Soil Sci. Plant. Anal., 18:1111-1116, 1987.

GREEN, R. N; TROWBRIDGE, R. L. e KLINKA, K. Towards a taxonomic classification of humus forms. For. Sci. Monogr., 29, 1993, p. 1-48.

JABIOL, B., ZANELLA, A., PONGE, J.-F.O., SARTORI, G., ENGLISCH, M., VAN DELFT, B., DE WAAL, R., LE BAYON, R.e.-C., 2013. A proposal for including humus forms in the World Reference Base for Soil Resources (WRB-FAO). Geoderma 192(0), 286-294.

KINDEL, A. A fragmentação Real: Heterogeinidade de remanescentes florestais e valor indicador das formas de húmus. Tese de Doutorado. Programa de Pós-Graduação em Geografia, 2001, Universidade Federal do Rio de Janeiro.

KINDEL, A. e GARAY, I. Caracterização de ecossistemas da Floresta Atlântica de Tabuleiros por meio das formas de húmus. Rev. Bras. de Ciências do Solo, 25, 2001, p. 551-563.

KINDEL, A.; GARAY, I. Humus form in ecosystems of the Atlantic Forest, Brazil. Geoderma, v. 108, n. 1-2, p. 101-118, 2002.

MCCUNE, B., MEFFORD, M.J., 1999. PC-ORD: multivariate analysis of ecological data. MjM Software Design. 
MYERS, N. Threatened biotas: Hotspots in tropical forests. The Environmentalist 8(3), 1988, p.1-20.

MYERS, N. The biodiversity challenge: Expanded hotspots analysis. The Environmentalist 10(4), 1990, p. 243256.

ODUM, E. P. Ecologia. Rio de Janeiro: Ed. Guanabara Koogan S. A., 1988.

PONGE, J. F. Humus forms in terrestrial ecosystems: a framework to biodiversity. Soil Biology and Biochemistry, v. 35, n. 7, p. 935-945, 2003.

PONGE, J. F. Plantesoil feedbacks mediated by humus forms: A review. Soil Biology \& Biochemistry 57 (2013) $1048 \mathrm{e} 1060$

PONGE, J.-F., CHEVALIER, R., 2006. Humus Index as an indicator of forest stand and soil properties. Forest Ecology and Management 233(1), 165-175.

REIS, A.; BECHARA, F. C.; ESPINDOLA, M. B.; VIEIRA, N. K.; SOUZA, L. L. Restauração de áreas degradadas: a nucleação como base para incrementar os processos sucessionais. Natureza \& Conservação, Curitiba, PR, v. 1, n. 1, p. 28-36, abr. 2003.

SOS Mata Atlântica e INPE. Atlas dos Remanescentes Florestais da Mata Atlântica. São Paulo, 2013.

SWIFT, M. J., HEAL, O. W. e ANDESON, J. M. Decomposition in Terrestrial Ecosystems. University of California Press, Berkley, Los Angeles, 1979.

TOUTAIN, F. 1981. Les humus forestiers. Structures et modes de fonctionnement. Revue Forestiere Francaise 33: 449-477.

ZANELlA, A., JABIOL, B., PONGE, J.F., SARTORI, G., DE WAAL, R., VAN DELFT, B., GRAEFE, U., COOLS, N., KATZENSTEINER, K., HAGER, H., ENGLISCH, M., 2011a. A European morpho-functional classification of humus forms. Geoderma In Press, Corrected Proof.

ZANELLA, A., JABIOL, B., PONGE, J.F., SARTORI, G., WAAL, R.D., DELFT, B.V., GRAEFE, U., COOLS, N., KATZENSTEINER, K., HAGER, H., ENGLISCH, M., BRETHES, A., BROLLK, G., GOBAT, J.M., BRUN, J.J., MILBERT, G., KOLB, E., WOLF, U., FRIZZERA, L., GALVAN, P., KOLLI, R., BARITZ, R., KEMMERSE, R., VACCA, A., SERrA, G., BANAS, D., GARlATO, A., S. CHERSiCH, KLIMO, E., LANGOHR, R., 2011 b. European humus forms: Base reference. 